\title{
A Telerobotic System for Augmentation of Endoscopic Surgery
}

\author{
Russell H. Taylor (1), Janez Funda (1), David LaRose (1), Michael Treat, MD (2) \\ (1) IBM T. J. Watson Research Center; Yorktown Heights, New York 10598 \\ Phone: (914)784-7796; Fax: (914)784-6282; Email: rht@watson.ibm.com \\ (2) College of Physicians \& Surgeons; Columbia University; N.Y., N. Y. 10032
}

\begin{abstract}
We have begun development of a telerobotic system to replace a human camera operator in endoscopic surgical procedures. The system will consist of a remote centerof-motion manipulator holding the endoscope; a computer workstation supporting image processing, graphics, manipulator control, and other functions; and a variety of man-machine interfaces (voice, instrumented joystick, etc.). A prototype system using an industrial robot has been implemented and used to demonstrate essential functions.
\end{abstract}

\section{Introduction}

Computer integrated surgical procedures (diagnosis, planning, execution, and followup) may be expected to increase dramatically in the coming decade, as present trends toward geometrically precise and minimally invasive surgery accelerate. A number of systems have been developed for various forms of "frameless" stereotactic neurosurgical procedures (e.g., [1], [2], [3]), orthopaedics (e.g., [4], [5]), craniofacial surgery ([6]), ENT (e.g., [7]) and other procedures. ' One common characteristic of these systems is that they rely on intraoperative position sensing to augment a human surgeon's ability to accurately execute a surgical plan based on 3D medical images. A machine capability is coupled with human judgement to perform a task better than either could do alone.

Similarly, the number of endoscopic and other minimally invasive surgical procedures has grown explosively over the past few years. ${ }^{2}$ Two salient characteristics of these procedures are that the surgeon cannot directly manipulate the patient's anatomy with his fingers and that he cannot directly observe what he is doing. Instead, he must rely on instruments that can be inserted through a trocar or through the working channel of an endoscope. Often, he must rely on an assistant to point the endoscopic camera while he performs the surgery. The awkwardness of this arrangement has led a number of researchers to develop robotic augmentation devices for endoscopic surgery (e.g., [10], [11], [12], [13]).

The key requirements of any telerobotic surgical aid are safety, responsiveness to the surgeon's desires, dexterity, and precision in executing the task. Furthermore, even early versions should offer real advantages to the human user while not requiring a fundamental

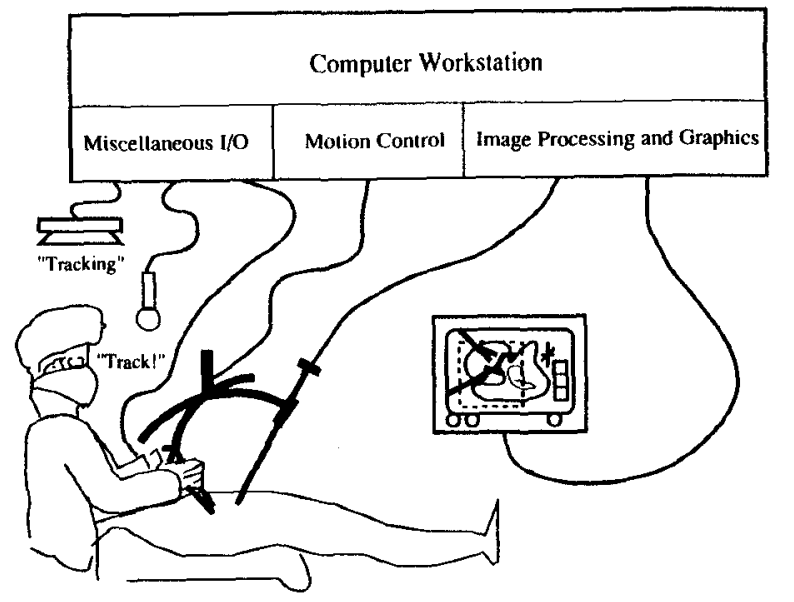

Figure 1. System Overview

1 This list is not exhaustive. A fuller literature summary may be found in [8] or in some of the other papers cited.

2 For example, about 20,000 laparoscopic cholecystectomies were performed in 1990; in 1993 the number is expected to be $600,000[9]$. 
change in other aspects of the surgical procedure. Consequently, we have chosen to concentrate initially on the relatively simple task of pointing the endoscopic camera. Even this task is non-trivial for human operators, especially when angled or flexible endoscopes are used, and it is plausible to expect that a computercontrolled device can do better than the average assistant. A telerobotic system would reduce the number of people required around the operating table and could increase the surgeon's direct control over an important aspect of the procedure. Further, the experience gained will permit ready extension to more delicate tasks.

\section{System Overview and Functions}

The system architecture is illustrated in Figure 1. The endoscope will be manipulated by a remote centerof-motion robot positioned so that its center-of-motion coincides with the entry point of the endoscope into the patient's body. The camera will be interfaced to a frame grabber/image processing system on a computer workstation, which will also control the manipulator, and will support a number of other man-machine interfaces, such as computer voice recognition, synthesized speech, joystick-like devices mounted on the surgeon's instruments, and similar devices

The computer is capable of visually tracking the surgeon's instruments and computing their $3 \mathrm{D}$ position relative to the position of the camera. In the simplest use

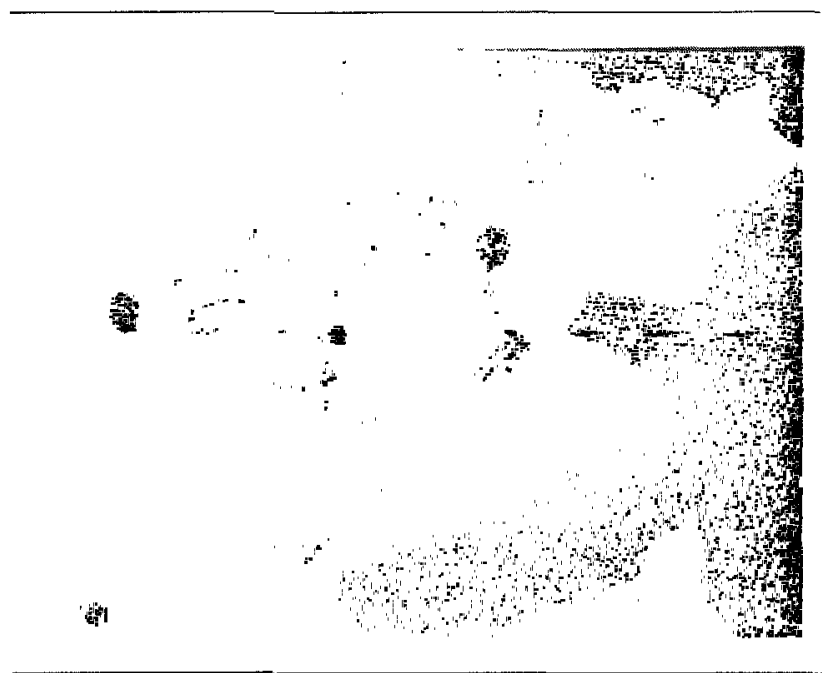

Figure 2. Remote Center-of-Motion Robot

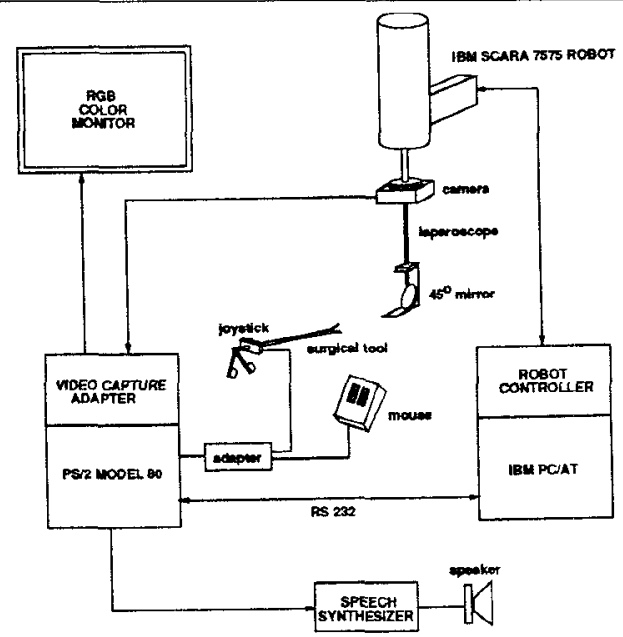

Figure 3. Experimental system.

of this facility, the surgeon can point to an anatomical feature and have the computer point the camera at it. Alternatively, the surgeon can use an instrumentmounted joystick to teleoperate the robot to position the camers or to position a cursor on the display screen to designate a feature. The computer can then aim the camera at a designated feature, can visually zero-in on a desired target, and can also visually servo to track the surgeon's instruments or anatomical features. Similarly, the surgeon can designate multiple points along a path, cause the manipulator to zoom in for a closer look, and then instruct the manipulator to slowly traverse the designated path while he concentrates his primary attention on the screen (e.g., to inspect along a blood vessel). Other functions include geometric calibration of the camera-lens system, image archival and retrieval functions for recording key scenes in surgery and recalling reference images, etc.

\section{Prototype Implementation and Status}

We are presently modifying a remote center-ofmotion manipulator built earlier for craniofacial surgery [8] by adding motors and a suitable camera carrier. An operational system is expected by Fall 1992. In the mean time, we have constructed the simple 4 degree-offreedom system shown in Figure 3, using an IBM 7576 robot, PS/2-based image processing system, and a surgical laparoscope with removable "periscope" optics. Except for image archival, we have used this system to 
demonstrate the basic functions described above for $0^{\circ}$ and $90^{\circ}$ viewing angles. We have also begun to experiment with stereo visualization and graphics and with the use of narrow-angle stereo to define 3D anatomical feature and instrument locations. The image processing system can follow instruments at about $9 \mathrm{~Hz}$, which is adequate, although significant improvements can be made. Further, even a "novice" user can quickly learn to use the system well enough to easily position the camera where desired. Although considerable work remains to be done, this early experience is very encouraging.

\section{Acknowledgements}

We wish to acknowledge the assistance of Nils Bruun, Bob Olyha, and other members of IBM Central Scientific Services for design, fabrication, and interfacing assistance of the remote center-of-motion manipulator. We also wish to thank Ted Selker and Joe Rutledge of IBM for key technology used in the instrument-mounted joystick.

\section{References}

[1] Patrick J. Kelly, Bruce A. Kall, Stephan Goerss, and Franklin Earnest, "Computer-assisted Stereotaxic laser Resection of Intra-Axial Brain Neoplasms," J. Neurosurg, pp. 427-439, March 1986.

M. A. Lewis and G. A. Bekey, "Automation and Robotics in Neurosurgery: Prospects and Problems," in Michael L. J. Apuzzo, MD, editor, Neurosurgery for the Third Millenium, American Asociation of Neurological Surgeons, Spring 1992 (to appear).

S. Lavallee, "A New System for Computer Assisted Neurosurgery," Proc. 11'th IEEE Engineering in Medicine and Biology Conf., pp. 926-927, Seattle, Nov 1989.

Russell H. Taylor, Howard. A. Paul, Brent D. Mittelstadt, William Hanson, Peter Kazanzides, Joel F. Zuhars, Edward Glassman, Bela L. Musits, William L. Bargar, and William Williamson, "An Image-based Robotic System for Hip Replacement Surgery," Journal of the Robotics Society of Japan, pp. 111-116, October 1990.
J. L. Garbini, R. G. Kaiura, J. A. Sidles, R. V. Larson, and F. A. Matson, "Robotic Instrumentation in Total Knee Arthroplasty," Proc. 33rd Annual Meeting, Orthopaedic Research Society, p. 413, San Francisco, January 1987.

[6] Russell H. Taylor, Court B. Cutting, Yong-yil Kim, Alan D. Kalvin, David Larose, Betsy Haddad, Deljou Khoramabadi, Marilyn Noz, Robert Olyha, Nils Bruun, and Dieter Grimm, "A Model-Based Optimal Planning and Execution System with Active Sensing and Passive Manipulation for Augmentation of Human Precision in Computer-Integrated Surgery," Proc. 1991 Int Symposium on Experimental Robotics, Toulouse, France: Springer-Verlag, June 25-27 1991.

[7] Ludwig Adams, Joachim M. Gilsbach, Werner Krybus, Dietrich Meyer-Ebrecht, Ralph Mosges, and Georg Schlondorff, "CAS - A Navigation Support for Surgery," 3d Imaging in Medicine, pp. 411-423, Springer-Verlag Berlin Heidelberg, 1990.

[8] Russell H. Taylor, Howard. A. Paul, Court B. Cutting, Brent Mittelstadt, William Hanson, Peter Kazanzides, Bela Musits, Yong-Yil Kim, Alan Kalvin, Betsy Haddad, Deljou Khoramabadi, and David LaRose, "Augmentation of Human Precision in Computer-Integrated Surgery," Innovation et Technologie en Biologie et Medicine, 1992. (invited paper for special issue on Computer Enhanced Surgery)

[9] Clinica, as cited by Dr. Phil Green in a talk given at 1990 Soc. Amer. Gastrointestinal Endoscopic Surgeons, Monterrey

[10] K. Ikuta, M. Tsukamoto, and S. Hirose, "Shape memory alloy servo actuator system with electric resistance feedback and application for active endoscopes," IEEE Robotics and Automation Conference, 1988.

[11] "Voice controlled flexible endoscope", videotape, R. Sturges, Carnegie Mellon University.

[12] R. H. Sturges and S. Laowattana, "A flexible tendoncontrolled device for endoscopy," Proc IEEE Robotics and Automation Conference, Sacramento, 1991.

[13] B. L. Davies, R. D. Hibberd, A. Timoney, and J. Wickham, "A Surgeon Robot for Prostatectomies," Proc. Fifth International Conference on Advanced Robotics, pp. 871-875, Pisa, June 1991. 